\title{
Chiral surface waves supported by biaxial hyperbolic metamaterials
}

\author{
Wen-Long Gao ${ }^{1,2}$, Feng-Zhou Fang ${ }^{1}$, Yong-Min Liu ${ }^{3,4}$ and Shuang Zhang ${ }^{1,2}$ \\ We discover a new kind of surface wave on biaxial hyperbolic metamaterial, which, in the k-space, connects the two diabolical points (or \\ conical singularities) of the equifrequency surface. Interestingly, the propagation of such surface wave is found to be sensitive to the \\ refractive index of the surrounding dielectric medium, showing a convex, concave or flat phase front when the refractive index is varied. \\ Furthermore, the surface wave shows an elliptically polarized state, in which helicity is dependent on the propagation direction. This \\ feature can be utilized for the spin-controllable excitation of surface waves, opening a gateway towards integrated photonic circuits with \\ reconfigurable functionalities.
}

Light: Science \& Applications (2015) 4, e328; doi:10.1038/Isa.2015.101; published online 25 September 2015

Keywords: metamaterial; spin orbital coupling; surface wave

\section{INTRODUCTION}

The existence of electromagnetic (EM) waves on two-dimensional interfaces has been extensively studied over the last several decades. The most widely studied surface wave is surface plasmonic polariton $(\mathrm{SPP})^{1-4}$, which normally exists at the interface between a noble metal and a dielectric. Because of the unique properties of strong field confinement and intensity enhancement, SPPs have been extensively explored in applications like super-resolution imaging ${ }^{5-7}$, biosensing ${ }^{8,9}$, wave guiding ${ }^{9-11}$ and photolithography ${ }^{12,13}$. Recently, it has been shown that by nanostructuring the metal surface, it is possible to modify the dispersion of SPPs or excite the SPPs in a prescribed manner ${ }^{14-19}$. It has also been shown that surface waves can exist at the interface between an anisotropic dielectric medium and isotropic dielectric medium when a certain relationship between the dielectric constants is satisfied. This type of surface wave, so-called Dyakonov waves $^{20,21}$, are loss free, in sharp contrast to SPPs, which suffer from Ohmic loss. Dyakonov-wave-like surface waves are also supported on layered hyperbolic metamaterials and on photonic crystals with enhanced angle range under the long-wavelength limit ${ }^{22,23}$.

Hyperbolic metamaterials, a special kind of anisotropic metamaterial whose dielectric tensor elements have mixed signs, have attracted growing attention recently because they support very large wave vectors. Their exotic features enable many intriguing applications, such as sub-wavelength imaging ${ }^{24-27}$, hyper-lens ${ }^{28-30}$ and enhanced spontaneous and thermal emissions ${ }^{31-33}$ that are infeasible with natural materials. While most studies have focused on uniaxial hyperbolic metamaterials, it has been shown recently that by introducing in-plane anisotropy, the quadratic degeneracy point splits into two diabolical points in the equifrequency surface, and conical diffraction with different topological features from a conventional biaxial media has been investigated $^{34}$. In this letter, we demonstrate the existence of a new kind of surface wave between a biaxial hyperbolic metamaterial (BHM) and an isotropic dielectric material. In contrast to extensively studied surface waves such as SPPs and Dyankonov waves, whose inplane wave vector is greater than that of the bulk modes, the in-plane wave vector of the surface wave supported by BHM lies between two bulk modes. Interestingly, the wavefront of the surface mode can be convex, concave or flat, by varying the refractive index of the surrounding dielectric medium. More remarkably, in general, the surface wave shows an elliptically polarized state, in which helicity is dependent on the propagation direction, leading to spin-controlled excitation of the surface wave.

\section{MATERIALS AND METHODS}

Without loss of generality, BHM's permittivity tensor can be written as $\operatorname{diag}\left[\varepsilon_{x}, \varepsilon_{y}, \varepsilon_{z}\right]$, with $\varepsilon_{z}$ being negative and $\varepsilon_{x}<\varepsilon_{y}$ being positive. Under Heaviside-Lorentz units, the general Fresnel equation for an equifrequency contour (EFC) can be expressed as

$$
\sum_{i=x, y, z} \frac{k_{i}^{2}}{k^{2}-\varepsilon_{i}}=1
$$

A typical EFC of a lossless biaxial hyperbolic metamaterial is shown in Figure 1a. There exist four singular points in the $k_{\mathrm{x}}-k_{\mathrm{z}}$ plane located at $\left[k_{D x}= \pm k_{0} \sqrt{\varepsilon_{z}\left(\varepsilon_{x}-\varepsilon_{y}\right) /\left(\varepsilon_{x}-\varepsilon_{z}\right)}, k_{D z}= \pm k_{0} \sqrt{\varepsilon_{x}\left(\varepsilon_{y}-\varepsilon_{z}\right) /\left(\varepsilon_{x}-\varepsilon_{z}\right)}\right]$. These singular points are reminiscent of the Dirac points for electrons extensively investigated in graphene, but in the momentum space. Next, we numerically solve for surface waves at the interface between the BHM and an isotropic dielectric material, which involves searching for hybrid modes composed of both transverse-electric and 


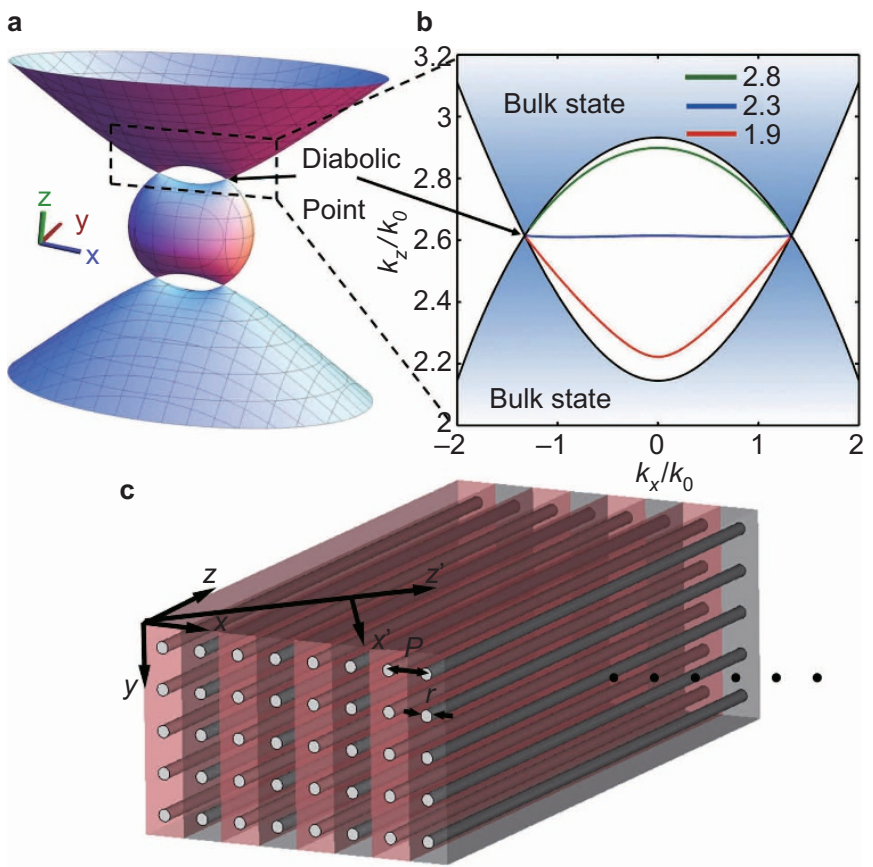

Figure 1. (a) 3D equi-frequency surface of a biaxial hyperbolic material. (b) On the interface between biaxial hyperbolic material and normal covering medium, an anomalous surface wave exists in the gap between degeneracy points. (c) Configuration of a realistic structure to realize $\mathrm{BHM}$. The $\mathrm{Si} / \mathrm{SiO}_{2}$ multilayer dielectric structure provides large in-plane anisotropy, while the embedded metallic nanowires lead to a negative permittivity along the $z$ direction. The unit cell of the BHM has dimensions $p_{x} \times p_{y}=120 \times 60 \mathrm{~nm} \times \mathrm{nm}$, which is a deep subwavelength to minimize the nonlocality of the system. The ratio of the $\mathrm{Si}$ and $\mathrm{SiO}_{2}$ segments are all 0.5 in a unit cell. The diameter of the Ag nano-wires is $18 \mathrm{~nm}$, which is less than the skin depth of silver.

transverse-magnetic (TE and TM) polarizations. The interface is in the $x-z$ plane, where $z$ represents the direction with negative permittivity and $x$ is the direction with the smaller permittivity tensor. Similar to Dyakonov waves, the surface wave supported at the surface of the BHM involves both eigenmodes inside the metamaterial and TE and TM waves in the surrounding dielectric medium. For ease of discussion, we define a local coordinate frame $\left[x^{\prime}, y, z^{\prime}\right]$ with $z^{\prime}$ defined to be along the propagation direction of the surface wave (Figure 1c). In this local frame, the components of the TM and TE mode in the surrounding dielectric medium with relative permittivity $\varepsilon_{s}$ can be expressed in the local frame as:

$$
E_{\mathrm{TM}}=\left[\begin{array}{c}
0 \\
q \\
i k_{\mathrm{TM}}
\end{array}\right] H_{\mathrm{TM}}=\left[\begin{array}{c}
-\varepsilon_{c} \\
0 \\
0
\end{array}\right] E_{\mathrm{TE}}=\left[\begin{array}{l}
1 \\
0 \\
0
\end{array}\right] H_{\mathrm{TE}}=\left[\begin{array}{c}
0 \\
q \\
i k_{\mathrm{TE}}
\end{array}\right]
$$

where $k_{\mathrm{TM}}$ and $k_{\mathrm{TE}}$ are the decay constants along the $y$ direction for the TM and TE mode, respectively, and $q$ is the wave vector along the $z^{\prime}$ direction. Components of the two eigenmodes inside the metamaterial can be numerically solved by substituting solutions of Equation (1) into Maxwell Equations. Finally, all the tangential $E$ and $H$ components of the four modes can be written into a characteristic matrix:

$$
\bar{F}=\left[\begin{array}{cccc}
0 & 1 & E_{x^{\prime}} & E_{x^{\prime}}^{\prime} \\
i k_{\mathrm{TM}} & 0 & E_{z^{\prime}} & E_{z^{\prime}}^{\prime} \\
-\varepsilon_{s} & 0 & H_{x^{\prime}} & H_{x^{\prime}}^{\prime} \\
0 & i k_{\mathrm{TE}} & H_{z^{\prime}} & H_{z^{\prime}}^{\prime}
\end{array}\right]
$$

Boundary conditions at the interface require that the tangential components of electric field and magnetic field are matched. In order to obtain the surface mode, we search through the $k_{x}$ and $k_{z}$ parameter space to find the existence of a nonzero solution by calculating the rank of the matrix $\bar{F}$. Specifically, the boundary condition at the corresponding point is perfectly matched when the rank of the matrix is reduced to 3 .

The dispersion of the surface waves for different refractive indices of the surrounding medium is shown in Figure 1b. Here, we set $\varepsilon_{x}=4.6$, $\varepsilon_{y}=8.6$ and $\varepsilon_{z}=-3.62$, which are retrieved by using the generalized Maxwell-Garnett theory ${ }^{35}$ from a realistic composite structure given in Figure 1c. The structure consists of a $\mathrm{Si} / \mathrm{SiO}_{2}$ multilayer medium (stacked along $x$ direction) with an array of metallic nanowires oriented along the $z$ direction embedded inside the layered medium. It was discovered that when the refractive index of the surrounding medium $\varepsilon_{\mathrm{s}}$ satisfies $\varepsilon_{x}<\varepsilon_{s}<\varepsilon_{y}$, a surface state exists whose equifrequency curve connects the two diabolical points. When $\varepsilon_{s}$ is increased, the equifrequency curve gradually evolves from a concave shape to a convex one. At $\varepsilon_{s} \approx 5.3$, the dispersion becomes flat, indicating that the surface wave can propagate without diffraction. An analytical solution of the effective index of the surface mode can be obtained for $k_{x}=0$, which gives the result: $k_{z}=k_{0} \sqrt{\varepsilon_{s} \varepsilon_{y}\left(\varepsilon_{s}-\varepsilon_{z}\right) /\left(\varepsilon_{s}^{2}-\varepsilon_{y} \varepsilon_{z}\right)}$. Because the EFCs of the surface waves always connect the diabolic points, then by setting $k_{z}=k_{D z}$, the condition for generating a flat dispersion can be obtained.

\section{RESULTS AND DISCUSSION}

To verify the effective medium description for the nanowire medium, we numerically solve the dispersion of the surface mode by using COMSOL. Both the real and imaginary parts of the mode index are shown in Figure 2. The numerically calculated mode index for the nanowire medium matches reasonably well with that which is obtained analytically by the effective medium approach, with a transition of the phase-front from concave to convex when the refractive index of the surrounding medium decreases. The slight deviations from the ideal effective medium case may be caused by nonlocalities ${ }^{36}$. As realistic metal parameters are used, there is ohmic loss in the system, leading to an imaginary part of the mode index, as shown in Figure $2 \mathrm{~b}$. However, the nanowire medium exhibits significantly lower optical loss than the effective medium because the effective medium formulism assumes a homogeneous distribution of the electric field inside the nanowire. Interestingly, for both effective medium and nanowire structure, the surface mode with a smaller refractive index $\varepsilon_{s}$ of the surrounding medium exhibits higher loss for both the effective medium and nanowire structure. This trend is due to the greater $z$ component of the electric field inside the hyperbolic medium with smaller $\varepsilon_{s}$.

To calculate the surface state in BHM, we carry out full-wave simulations at a wavelength of $1550 \mathrm{~nm}$ with both the homogenous effective medium (Figure $3 \mathrm{a}, 3 \mathrm{~b}$ and $3 \mathrm{c}$ ) and the realistic metamaterial structure (Figure $3 \mathrm{~d}, 3 \mathrm{e}$ and $3 \mathrm{f}$ ). In the effective medium simulation, no loss is included in the effective parameters. In the simulation, the surface waves are excited by a linearly polarized Gaussian wave incident along the $y$-direction at one side, with the centre of the Gaussian beam aligned with the interface. The $y$ components of the electric fields for different refractive indices of the surrounding medium are shown in Figure 3, exhibiting flat, convex and concave wavefronts at $n_{s}=2.3$, 1.9 and 2.8, respectively (Figure $3 \mathrm{a}, 3 \mathrm{~b}$ and $3 \mathrm{c}$ ). The field pattern (Figure 3d, 3e and 3f) of the realistic metamaterial structure shows very similar features to that of the effective medium, in spite of the 

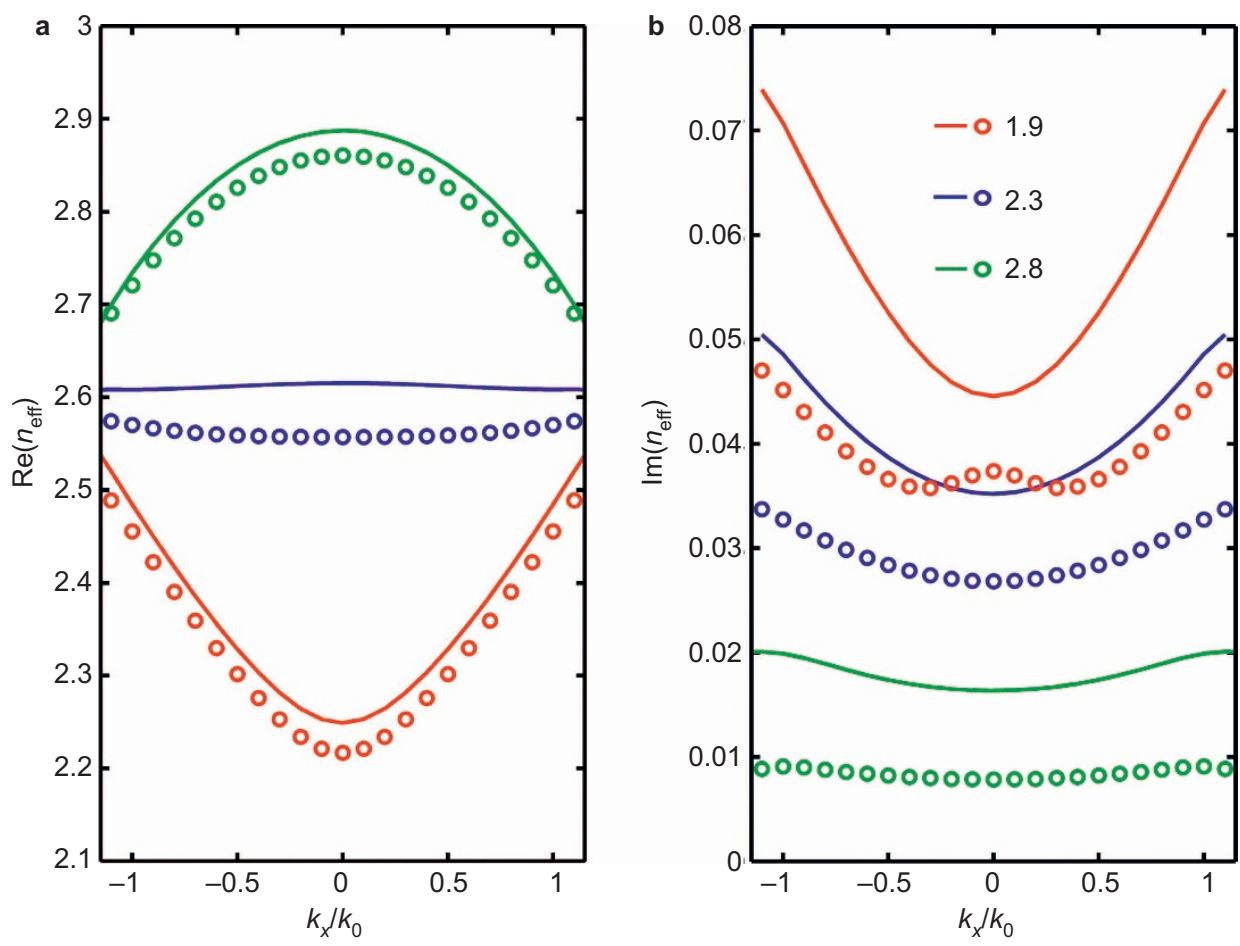

Figure 2. Real (a) and imaginary part (b) of mode indexes for different $n_{s}$. Results for effective medium and real structures are shown in solid lines and circles, respectively.

intensity attenuation along the propagation direction due to the Ohmic loss of the metal nanowires. The simulation results of the propagation of the surface wave in both the effective medium and realistic structures agree very well with the equifrequency curve shown in Figure 1b. Here, the diffractionless propagation of the surface wave is a close analogue to spatial solitons in nonlinear optics, but is purely based on linear properties of the biaxial metamaterial and the surrounding dielectric and therefore suitable for wave guiding at low optical intensities. In addition, the sensitivity of the wavefront to the refractive index of the surrounding medium may lead to the application of the BHM as a refractive index sensor. An estimation of the coupling efficiency is obtained by numerically integrating the power of surface waves. For the effective medium, the coupling efficiency for a $y$-polarized incident wave is $39.7 \%, 46.3 \%$ and $48.5 \%$ for $n_{s}=1.9,2.3$ and 2.8 , respectively. The coupling efficiency for the nanowire medium is numerically calculated to be $32.5 \%$ at $n_{s}=1.9$, which is slightly lower than that of the effective medium.

The polarization state of the surface mode is obtained from the nonzero solution of the aforementioned characteristic matrix. From the field basis used in matrix $\bar{F}$, we can express the total transverse field components in $x^{\prime}$ and $y$ directions in the surrounding medium and BHM as $\left[i \gamma, k_{z^{\prime}}\right]$ and $\left[i \gamma, \varepsilon_{y} k_{z^{\prime}}\right]$, respectively. Here, $k_{z^{\prime}}=\sqrt{k_{x}^{2}+k_{z}^{2}}$ and $i \gamma$ is the ratio between the coefficients of TE and TM modes obtained from the nonzero solution of matrix $\bar{F}$. The plot of $\gamma v s k_{x}$ is shown in Figure $4 \mathrm{a}$. For $k_{x}=0$, the surface wave is purely TM-polarized; thus, the amplitude of TE is zero. Interestingly, the phase difference between the TE and TM components is constantly $\pi / 2$ for positive $k_{x}$ and $-\pi / 2$ for negative $k_{x}$. This means that the electric field in the surrounding medium is elliptically polarized, with both the sign and magnitude of the ellipticity dependent on $k_{x}$ as shown in Figure $4 \mathrm{~b}$ $4 \mathrm{j}$. The polarization state of the surface wave inside the BHM preserves the same handedness as in the surrounding medium due to the boundary condition across the interface.

To understand the $\pi / 2$ phase difference between the TE and TM components of the surface wave, we write, in the local coordinate, the three components of the Poynting vector in the surrounding medium as

$$
\begin{gathered}
S_{x^{\prime}}=-\gamma k_{\mathrm{TM}} q \\
S_{y^{\prime}}=\frac{1}{2}\left(-i|\gamma|^{2} k_{\mathrm{TM}}+i k_{\mathrm{TM}} \varepsilon_{s}\right) \\
S_{z^{\prime}}=\frac{1}{2}\left(|\gamma|^{2} q+\varepsilon_{s} q\right)
\end{gathered}
$$

In the above equations, the $y^{\prime}$ and $z^{\prime}$ components of the Poyting vector are imaginary and real, respectively, regardless of the value of $\gamma$. However, to ensure that the $x^{\prime}$ component of Poynting vector in the surrounding medium is real, $\gamma$ needs to be real, i.e., there is a $\pi / 2$ phase difference between TE and TM components. Further, we write the angle between phase velocity and group velocity as

$$
\theta=\tan ^{-1}\left(S_{x^{\prime}} / S_{z^{\prime}}\right)=\tan ^{-1}\left(\frac{2|\gamma| k_{\mathrm{TM}}}{|\gamma|^{2}+\varepsilon_{s}}\right)
$$

Intuitively, the angle between the phase velocity and the group velocity increases when the equifrequency contour of the surface wave deviates from a circular shape. Thus, $\theta$ decreases with the increase in $\varepsilon_{s}$ as the EFC varies from concave to convex and approaches a circular shape. On the other hand, the ratio between the TE and TM components increases monotonously with $\theta$ for $\gamma$ less than $\sqrt{\varepsilon_{s}}$, as indicated by Equation (4). That is to say, a concave surface wave exhibits smaller ellipticity than the flat and concave surface waves. 

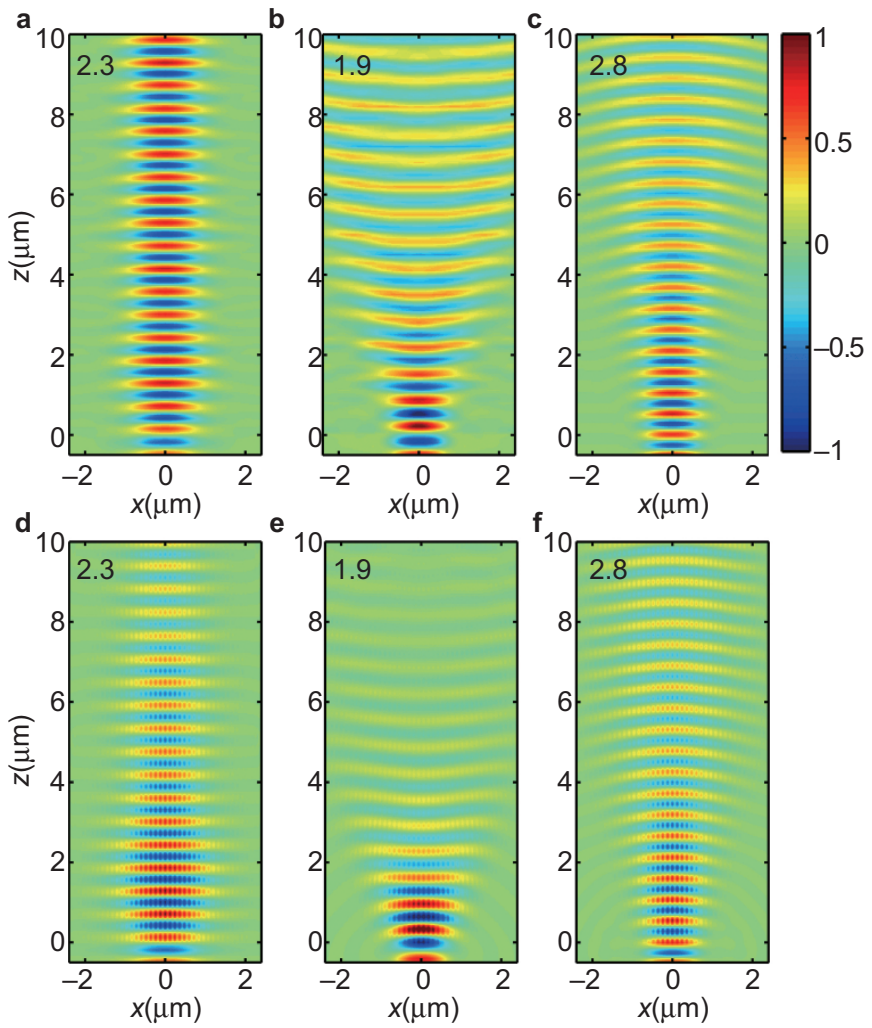

Figure 3. The flat, concave and convex wave-fronts of the surface wave supported by a biaxial hyperbolic metamaterial. (a, b, c) Simulation of the field distribution $\left(E_{y}\right)$ of the surface wave at the interface between an isotropic dielectric medium and the biaxial hyperbolic effective medium. The refractive indices of surrounding medium for (a), (b) and (c) are $n_{c}=2.3,1.9$ and 2.8, respectively. (d, e, f) The same as $(\mathbf{a}, \mathbf{b}, \mathbf{c})$, but with the effective medium replaced by the realistic metamaterial structures.

Interestingly, in the simulation of the excitation of the surface wave, we observe very strong helicity-dependent directional surface wave excitation for the concave surface wave. Specifically, an incident light with circular polarization can preferably excite the surface wave along one specific direction or the other depending on the helicity of the incident wave. This arises from the direction-dependent helicity of the surface wave, which is verified by the full-wave simulation as shown in Figure 5. The surface wave is tilted to the right for the left circularly polarized (LCP) incidence, or to the left for the right circularly polarized (RCP) incidence, due to the matching of the helicity between the incident wave and the surface wave of oblique directions.

\section{CONCLUSION}

In conclusion, we demonstrate a new kind of surface wave between a biaxial hyperbolic metamaterial and a normal isotropic dielectric. It is shown that the wave front of the surface wave is sensitively dependent on the refractive index of the surrounding medium. Almost flat $k$ dispersion exists for certain refractive indexes of the surrounding medium, which could lead to highly compact self-guiding surface waves without adding nonlinearity or lateral confinements. The surface wave supported by a BHM shows an elliptically polarized state, whose ellipticity depends on the direction of propagation. Therefore, strong spin-orbital coupling is achieved in our system, which allows us to selectively control the wave propagation by the polarization handedness. These findings expand the horizon of nano optics based a

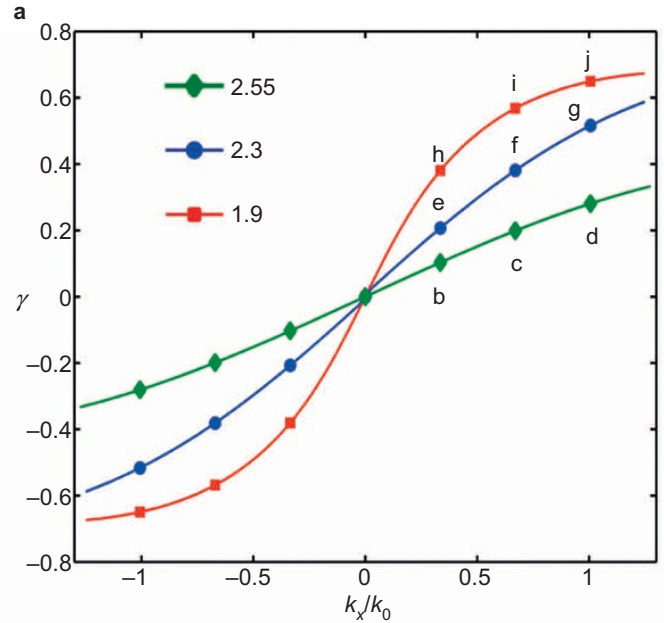

b
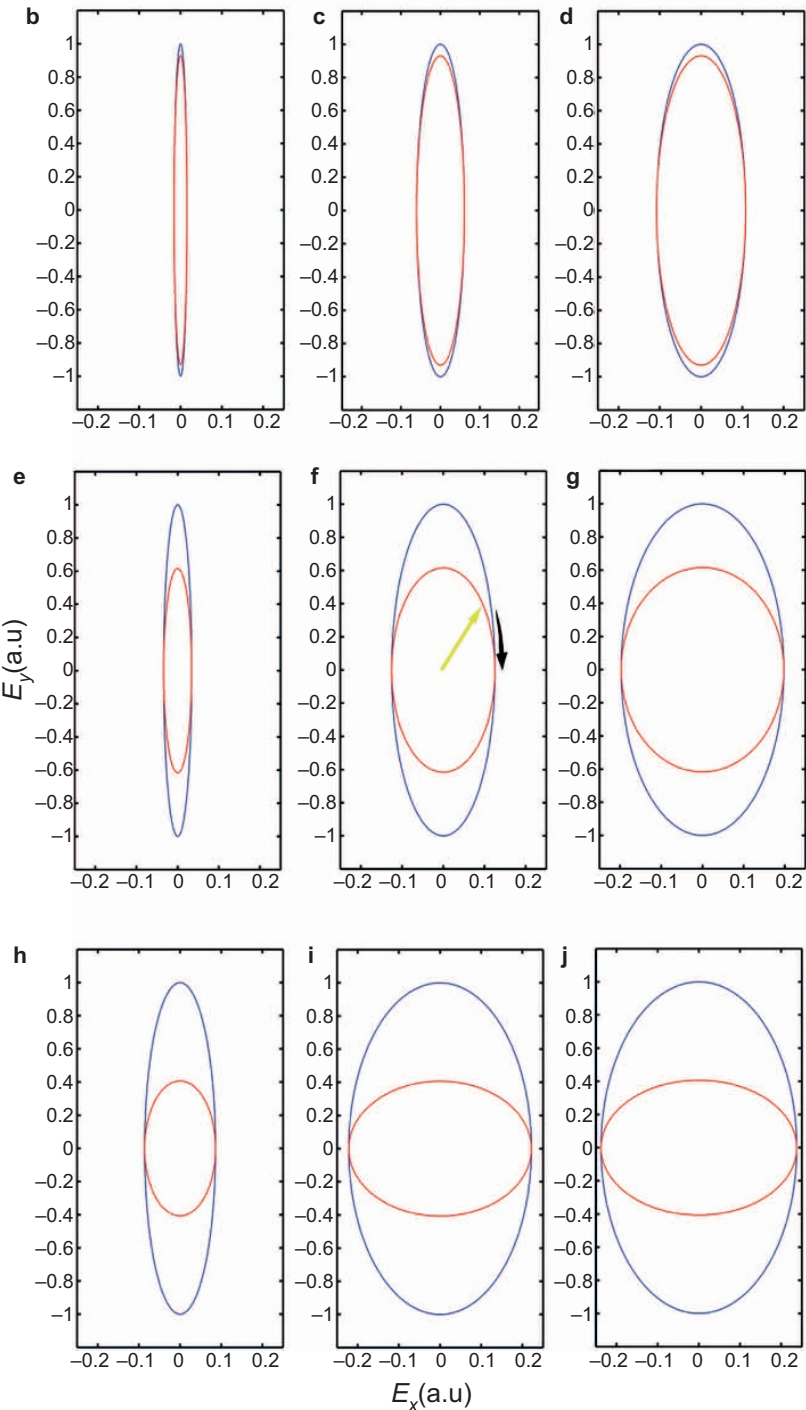

Figure 4. (a) The ratio of the amplitude of the TE to TM mode in surrounding medium. The phase difference between the two modes is $\pm \pi / 2$, leading to an elliptically polarized mode for nonzero $k_{x}$. The red, blue and green curves correspond to $n_{s}=1.9$ (concave), 2.3 (flat) and 2.8 (convex), respectively. (b-j) Polarization in both surrounding medium (red) and BHM (blue) under different $k_{x}$ and $n_{s}$. For positive (negative) $k_{x}$, surface waves show right (left) handedness elliptical polarization. 

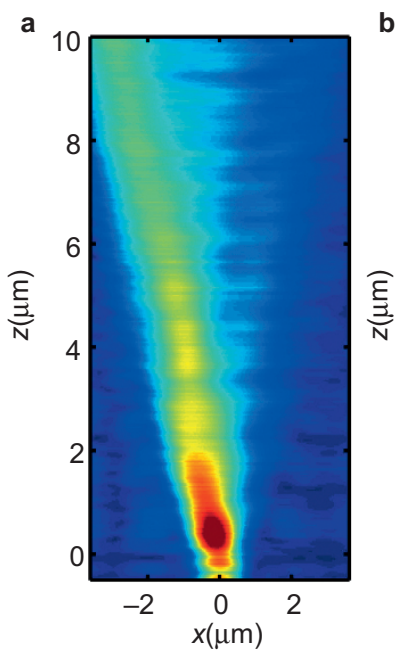

\section{b 10}

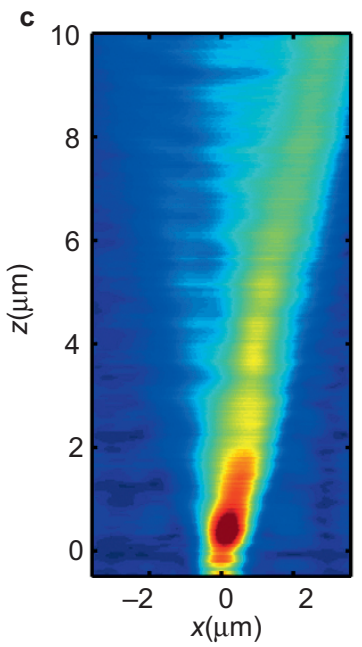

d
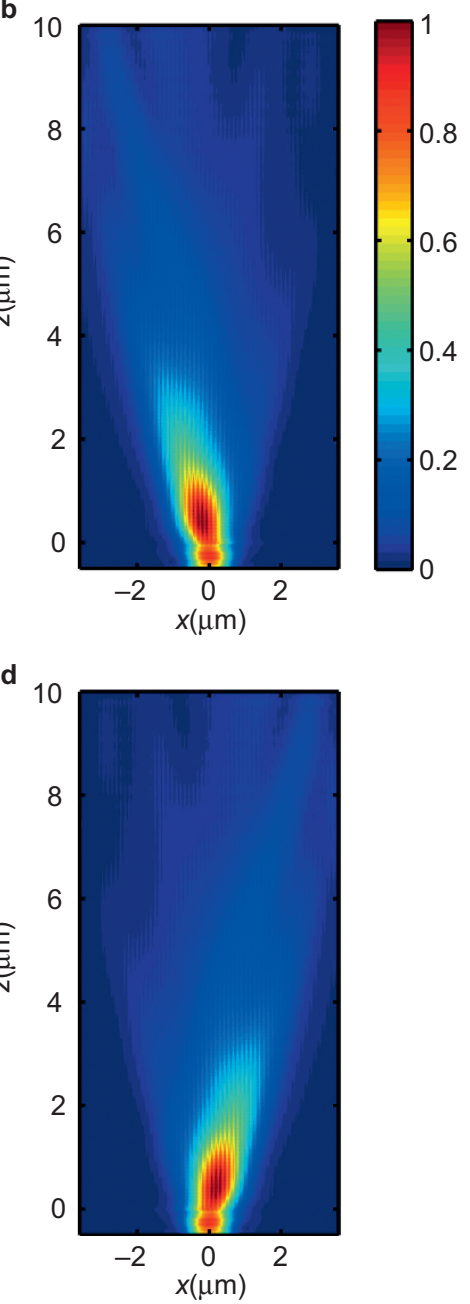

Figure 5. The simulation results of normalized electric field intensity under LCP excitation (a, b) and RCP excitation (c, d). The excited surface wave is tilted right and left for LCP and RCP, respectively. (a) and (c) show simulations with effective medium, while (b) and (d) show simulation results with realistic metamaterial structures.

on surface waves, manifesting potential applications in both classical and quantum optical signal communication and processing.

\section{ACKNOWLEDGEMENTS}

This work is supported by NSFC under Project 61328503 and the Leverhulme Trust (Grant No. RPG-2012-674). W. G thanks Chinese Scholarship Council Grant No. 201306250106.

1 Raether H. Surface Plasmons on Smooth and Rough Surfaces and on Gratings. Berlin: Springer-Verlag; 1988.

2 Ozbay E. Plasmonics: merging photonics and electronics at nanoscale dimensions. Science 2006; 311: 189-193.

3 Polman A, Atwater HA. Plasmonics: optics at the nanoscale. Mater Today2005; 8: 56

4 Tsakmakidis KL, Hermann C, Klaedtke A, Jamois C, Hess O. Surface plasmon polaritons in generalized slab heterostructures with negative permittivity and permeability. Phys Rev B 2006; 73: 085104.

5 Pendry JB. Negative refraction makes a perfect lens. Phys Rev Lett 2000; 85: 39663969

6 Fang N, Lee H, Sun C, Zhang X. Sub-diffraction-limited optical imaging with a silver superlens. Science 2005; 308: 534-537.

7 Taubner T, Korobkin D, Urzhumov Y, Shvets G, Hillenbrand R. Near-field microscopy through a SiC superlens. Science 2006; 313: 1595.

8 Anker JN, Hall WP, Lyandres O, Shah NC, Zhao J et al. Biosensing with plasmonic nanosensors. Nat Mater 2008; 7: 442-453.

9 Lal S, Link S, Halas NJ. Nano-optics from sensing to waveguiding. Nat Photonics 2007; 1: 641-648.

10 Maier SA, Kik PG, Atwater HA, Meltzer S, Harel E et al. Local detection of electromagnetic energy transport below the diffraction limit in metal nanoparticle plasmon waveguides. Nat Mater 2003; 2: 229-232.

11 Bozhevolnyi SI, Volkov VS, Devaux E, Laluet JY, Ebbesen TW. Channel plasmon subwavelength waveguide components including interferometers and ring resonators. Nature 2006; 440: 508-511.

12 Srituravanich W, Fang N, Sun C, Luo Q, Zhang X. Plasmonic nanolithography. Nano Lett 2004; 4: 1085-1088.

13 Liu Z, Steele JM, Srituravanich W, Pikus Y, Sun C et al. Focusing surface plasmons with a plasmonic lens. Nano Lett 2005; 5: 1726-1729.

14 Pendry JB, Martín-Moreno L, Garcia-Vidal FJ. Mimicking surface plasmons with structured surfaces. Science 2004; 305: 847-848.

15 Hibbins AP, Evans BR, Sambles JR. Experimental verification of designer surface plasmons. Science 2005; 308: 670-672.

16 Williams CR, Andrews SR, Maier SA, Fernández-Domínguez AI, Martín-Moreno L et al. Highly confined guiding of terahertz surface plasmon polaritons on structured metal surfaces. Nat Photonics 2008; 2: 175-179.

17 Gan Q, Fu Z, Ding YJ, Bartoli FJ. Ultrawide-bandwidth slow-light system based on $\mathrm{THz}$ plasmonic graded metallic grating structures. Phys Rev Lett 2008; 100: 256803.

18 Huang $L$, Chen X, Bai B, Tan Q, Jin G et al. Helicity dependent directional surface plasmon polariton excitation using a metasurface with interfacial phase discontinuity. Light Sci App/ 2013; 2: e70; doi:10.1038/lsa.2013.26.

19 Lin J, Mueller JP, Wang Q, Yuan G, Antoniou N et al. Polarization-controlled tunable directional coupling of surface plasmon polaritons. Science 2013; 340: 331-334.

20 D'yakonov MI. New type of electromagnetic wave propagating at an interface. Sov Phys JETP 1988; 67: 714-716.

21 Artigas D, Torner L. Dyakonov surface waves in photonic metamaterials. Phys Rev Lett 2005; 94: 013901.

22 Zapata-Rodríguez CJ, Miret JJ, Vuković S, Belić MR. Engineered surface waves in hyperbolic metamaterials. Opt Express 2013; 21: 19113-19127.

23 Jacob Z, Narimanov EE. Optical hyperspace for plasmons: Dyakonov states in metamaterials. Appl Phys Lett 2008; 93: 221109.

24 Jacob Z, Alekseyev LV, Narimanov E. Optical hyperlens: far-field imaging beyond the diffraction limit.Opt Express 2006; 14: 8247-8256.

25 Salandrino A, Engheta N. Far-field subdiffraction optical microscopy using metamaterial crystals: theory and simulations, Phys Rev B 2006; 74: 075103.

26 Shvets G, Trendafilov S, Pendry JB, Sarychev A. Guiding, focusing, and sensing on the subwavelength scale using metallic wire arrays. Phys Rev Lett 2007; 99: 053903.

27 Belov PA, Hao Y. Subwavelength imaging at optical frequencies using a transmission device formed by a periodic layered metal-dielectric structure operating in the canalization regime. Phys Rev B 2006; 73: 113110.

28 Liu Z, Lee H, Xiong Y, Sun C, Zhang X. Far-field optical hyperlens magnifying subdiffraction-limited objects. Science 2007; 315: 1686.

29 Smolyaninov II, Hung YJ, Davis CC. Magnifying superlens in the visible frequency range. Science 2007; 315: 1699-1701.

30 Rho J, Ye Z, Xiong Y, Yin X, Liu Z et al. Spherical hyperlens for two-dimensional sub-diffractional imaging at visible frequencies. Nat comm 2010; 1: 143.

31 Jacob Z, Smolyaninov II, Narimanov EE. Broadband Purcell effect: radiative decay engineering with metamaterials. Appl Phys Lett 2012; 100: 181105.

32 Tumkur T, Zhu G, Black P, Barnakov YA, Bonner CE et al. Control of spontaneous emission in a volume of functionalized hyperbolic metamaterial. Appl Phys Lett2011; 99: 151115.

33 Lu D, Kan JJ, Fullerton EE, Liu Z. Enhancing spontaneous emission rates of molecules using nanopatterned multilayer hyperbolic metamaterials. Nat Nanotech 2014; 9: 48-53.

34 Ballantine KE, Donegan JF, Eastham PR. Conical diffraction and the dispersion surface of hyperbolic metamaterials. Phys Rev A 2014; 90: 013803.

35 Sigvola $\mathrm{AH}$. Electromagnetic Mixing Formulas and Applications. London: Institution of Electrical Engineers; 1999.

36 Zapata-Rodríguez CJ, Miret JJ, Vuković S, Belić MR. Engineered surface waves in hyperbolic metamaterials. Opt Express 2013; 21: 19113-19127.

(c) This license allows readers to copy, distribute and transmit the Contribution as long as it is attributed back to the author. Readers are permitted to alter, transform or build upon the Contribution, and to use the article for commercial purposes. Please read the full license for further details at http://creativecommons.org/licenses/by/4.0/ 